Meta

Journal des traducteurs

Translators' Journal

\title{
La méthode du discours : termes, mots, expressions
}

\section{Henri Dumas}

Volume 22, numéro 2, juin 1977

URI : https://id.erudit.org/iderudit/002675ar

DOI : https://doi.org/10.7202/002675ar

Aller au sommaire du numéro

Éditeur(s)

Les Presses de l'Université de Montréal

ISSN

0026-0452 (imprimé)

1492-1421 (numérique)

Découvrir la revue

Citer cet article

Dumas, H. (1977). La méthode du discours : termes, mots, expressions. Meta, 22(2), 110-116. https://doi.org/10.7202/002675ar d'utilisation que vous pouvez consulter en ligne.

https://apropos.erudit.org/fr/usagers/politique-dutilisation/ 


\section{La méthode du discours: termes, mots, expressions}

《Terme» et «mot» sont en général donnés comme synonymes par les dictionnaires. Nous avons souvent l'impression qu'entre « terme » et « expression », il n'y a qu'une nuance, parfois imperceptible. Il serait bon que le terminologue, qui doit s'occuper de termes, bien sûr, sache à quoi s'en tenir. Sinon, il risque d'avoir des doutes sur sa propre identité, ce qui est toujours fâcheux. Il pourrait se demander, par exemple, s'il n'est pas un motard sédentaire ou un expressionniste qui s'ignore.

Trouver, c'est savoir où chercher. Sans démarches ni détours inutiles, nous consultons donc le grand maître en matière de lexicographie, Littré. Dans le corps de l'article «terme»du Littré, nous trouvons :

Termes, mots, expressions. Les termes sont distingués des mots en ce que ces derniers sont de la langue, et que les premiers sont du sujet, ainsi que les expressions sont de la pensée. "L'usage décide des mots, la convenance avec les choses fait la bonté des termes, le tour fait le mérite de l'expression», Laveaux. Termes propres. [...] Les termes propres sont ceux que l'usage a consacrés, pour rendre précisément les idées que l'on veut exprimer.

Admirons, au passage, l'élégance du style, la clarté, la précision, la concision. Tout est mis à sa place, les frontières bien indiquées. Nous savons déjà que le terminologue devra, comme tout un chacun, utiliser les mots qui existent dans la ou les langues où il exerce son art. Il devra désigner par les termes propres dans une langue, les sujets dont traitent ceux qui font connaître leur pensée dans une autre langue. Le terminologue, laissant au traducteur la responsabilité du style, du tour, lui indiquera, pour chaque chose, quel est le terme qui convient, en respectant, dans le choix de ses mots, l'usage. Grâce à lui, le traducteur pourra exprimer les idées d'un auteur avec autant de précision que l'auteur lui-même.

Nous avons donc maintenant, grâce à ces quelques phrases lapidaires, une notion claire et précise de ce que sont mots, termes et expressions. Pour en avoir une notion concrète, qui nous permette de tirer des conclusions pratiques, il nous faut des exemples.

Soit à exprimer l'idée d'un «grand mammifère à crinière, plus grand que l'âne, domestiqué par l'homme comme animal de trait et de transport ». Ce que nous venons, pour ce faire, d'écrire entre guillemets, constitue une expression. 
Cette expression est précise, car elle décrit bien l'idée que nous voulions transmettre, mais elle est longue et lourde.

Ce n'est pas la seule expression possible dans ce cas. Parmi toutes celles que nous pourrions imaginer, il en est une qui nous semble particulièrement remarquable, c'est l'expression «cheval». Pour ce que nous voulons dire en effet, cette dernière est au moins aussi précise que la précédente, puisqu'il nous suffira de consulter la rubrique «cheval » du Petit Robert pour 1'y retrouver. Elle est même plus précise, car nous pouvons nous référer à des chapitres ou articles de traités ou d'encyclopédies, voire à des ouvrages spécialisés, consacrés au cheval. En fait, cette expression nous permet, si nous le voulons, d'avoir accès à tout ce qui a été écrit en français au sujet de l'idée mentionnée plus haut. Cette expression est évidemment beaucoup plus courte et plus légère que la première.

On reconnaîtra sans peine, dans une telle expression, précise et concise, appliquée à un sujet, un terme, tel qu'il est défini par Littré.

Nous avons vu que «cheval»se trouve dans les dictionnaires de la langue française, qu'il est connu de ceux qui parlent notre langue et utilisé par eux. C'est donc un mot. D'ailleurs, le Petit Robert nous donne une excellente définition incidente du « mot (écrit) »:

... (sauf dans la langue écrite : suite de signes non interrompue).

《Cheval» est-il le seul terme dont nous disposons pour désigner un « grand mammifère à crinière... »? Non, nous connaissons aussi «canasson », qui est à peine plus long et " rosse », qui a l'avantage d'être plus court. Sont-ils aussi précis ? Voyons « canasson».

Populaire. Cheval et (spécialement) mauvais cheval, rosse. (Petit Robert)

"Canasson» transmet donc bien la même idée que «cheval», mais ce terme ajoute d'autres éléments d'information que «cheval» ne porte pas : « moi qui vous parle, je fais partie du groupe de ceux qui ne se soucient pas trop de la qualité de leur langage et je m'adresse aux membres de ce groupe, ou bien, je veux parler d'un cheval, soit, mais d'un mauvais cheval. » Pour désigner le « grand mammifère à crinière... » que nous avons enfourché au début de cet article nous avons bien trois termes, mais seul «cheval » est le terme propre. De même, «canasson » est le terme propre pour désigner « en langage populaire, un cheval et spécialement un mauvais cheval »; et « rosse » est le terme propre pour désigner un « mauvais cheval ».

Nous constatons qu'en français, mises à part les variantes orthographiques (container et contencur), il y a très peu de vrais synonymes. Ceci peut faire pleurer certains sur la prétendue pauvreté de notre vocabulaire. Que ces esprits chagrins se consolent : le vocabulaire français est en fait très économe et par conséquent très efficace, ce qui contribue à la richesse de la langue. Les linguistes nous expliqueraient sans doute qu'il en est ainsi parce que notre langue a été façonnée par un peuple de gens économes qui, dès qu'ils ont à leur disposition un mot qui risque de faire double emploi et d'être inutile, en profitent pour lui confier 
une nouvelle tâche, lui faire porter un nouveau sens ou préciser une nuance délicate. Peu nous chaut. Nous constatons simplement, nous félicitons de la richesse bien méritée de notre langue et faisons bien attention de ne pas utiliser un mot pour un autre.

Un mot, à condition qu'il corresponde à un sens, c'est-à-dire qu'il remplisse une fonction sémantique et non pas uniquement grammaticale, est donc un terme, et par suite une expression. Mais l'inverse n'est pas vrai, nous avons vu que toute expression n'est pas un terme et nous allons montrer qu'il existe des termes comportant plusieurs mots.

Soit à exprimer l'idée d'un « appareil de levage utilisé pour déplacer verticalement une charge sur une course limitée, actionné par de l'air comprimé agissant sur un piston transmettant directement son mouvement à la charge, sans l'intermédiaire d'un treuil ». Nul ne contestera que cette expression, pour être précise, n'en est pas moins lourde et encombrante. Mais nous ne pouvons pas la condenser en un seul mot, comme nous avions fait pour le " grand mammifère à crinière... 》. Le mieux que nous pouvons faire, c'est d'écrire « palan pneumatique à piston ». Cette dernière expression, désignant un sujet de façon précise et concise, est un terme. Or nous constatons que ce terme est constitué de quatre mots : trois mots sémantiques (palan, pneumatique, piston), dont chacun est un terme, et un mot grammatical (à).

Donc, un terme est une expression précise et concise désignant une notion (c'est-à-dire l'idée que nous nous faisons d'un concept) pouvant être utilisée dans le discours pour désigner, efficacement et économiquement, cette notion chaque fois que nous voudrons l'exprimer. Un terme se compose d'un mot ou de plusieurs mots rangés dans un certain ordre suivant les règles de la syntaxe. D'une façon tout à fait analogue, mais seulement analogue, un mot se compose d'un ou de plusieurs signes (lettres, accentuées ou non, traits d'union, apostrophes) rangés dans un certain ordre, sans espaces vides, suivant les règles de l'orthographe.

Cette analogie et le fait que, souvent, mot et terme se présentent exactement sous la même forme sont à l'origine de la confusion qui peut sembler exister entre l'un et l'autre.

Nous disposons donc des éléments de construction du discours : briques, panneaux préfabriqués, pans de mur. Il ne nous reste plus qu'à mettre au travail et au pied du mur (sans prendre cette expression au pied de la lettre) les différents corps de métier. Le seul qui nous intéresse ici, est celui des terminologues. Si nous voulons que nos vaches soient bien gardées, nos mots bien pesés, nos termes bien propres et nos expressions bien tournées, il convient que chacun fasse son métier et que chacun sache quel est son métier.

Le terminologue doit-il s'occuper de mots? Non, car ceux-ci sont l'affaire des lexicographes. Les mots se rencontrent dans les dictionnaires, au terminologue de savoir les y trouver. Bien sûr, certains mots nouveaux, qui existent car ils servent, n'ont pas encore eu le temps de se faire connaître, reconnaître et accepter. S'il a besoin de ces mots nouveaux, le terminologue doit savoir comment et où les 
chercher ; il doit aussi et surtout pouvoir faire la critique de ses sources d'information. Qu'il sache qu'en ce faisant, il œuvre en tant que lexicographe et ne fait pas son vrai métier de terminologue. Heureusement, pour une langue efficace, économique et propre comme le français, ces cas sont extrêmement rares et le terminologue peut, le plus souvent, se tirer d'affaire à l'aide des dictionnaires. Sa consigne est : n'utiliser que des mots existants, de préférence reconnus par les dictionnaires.

Doit-il s'occuper des expressions? S'il le fait, il devra recenser toutes les expressions possibles dans un domaine considéré, puisqu'il n'existe pas de recueils d'expressions, comme les dictionnaires pour les mots. Mission impossible. Point n'est besoin de nous abandonner aux délices de l'analyse combinatoire pour nous convaincre que les expressions possibles dans un domaine donné sont pratiquement innombrables. Non, c'est au rédacteur (auteur ou traducteur) qu'il appartient, pour chaque idée à transmettre, de construire l'expression juste, de tour élégant, de forme originale, pour la décrire. Le rédacteur bâtira ses expressions à l'aide des éléments préfabriqués (répondant exactement à ses spécifications) que lui fournira le terminologue. Ces éléments préfabriqués, ce sont, nous l'avons déjà vu, les termes.

Le terminologue doit donc se contenter de mettre à la disposition des autres corps de métier les termes dont ils ont besoin. C'est déjà une lourde tâche. Dans son accomplissement, il aura à résoudre deux problèmes : reconnaître les termes, les trouver.

Tout terme est une expression, mais toute expression n'est pas un terme. Comment reconnaître alors, si une expression constitue un terme ? Étant donné une expression, si nous en connaissons une autre, aussi précise et plus concise, pour décrire la même idée, l'expression donnée n'est pas un terme. Ceci ne veut pas dire que toute expression qui ne peut être réduite à une autre aussi précise et plus concise, constitue un terme. Souvenez-vous de la définition de Littré: «... et que les premiers (les termes) sont du sujet, ainsi que les expressions sont de la pensée ». Si l'expression décrit un sujet unique bien déterminé, il s'agit effectivement d'un terme. Si la pensée exprimée combine plusieurs sujets, il ne s'agit pas d'un terme mais d'une expression formée de plusieurs termes.

Il est des expressions qu'on retrouve souvent, chaque fois sous la même forme : ce sont les expressions consacrées. Celles-ci peuvent se présenter sous la forme d'une proposition ou d'une phrase ; elles sont alors du ressort du rédacteur qui, seul, peut décider s'il convient ou non d'employer cette expression consacrée, ou du traducteur qui, seul, peut proposer une expression équivalente dans une autre langue. Elles peuvent aussi se présenter sous la forme d'une association de mots et de termes qui, dans le discours, jouera exactement le même rôle qu'un terme. Il est souvent difficile, dans ce cas, de savoir s'il s'agit d'une expression consacrée ou d'un terme, et c'est bicn dommage, car il peut en résulter des travaux inutiles. Une «ficelle du métier» consiste à appliquer dans ce cas l'« essai de traduction ». $\mathrm{Si}$, en traduisant mot à mot une expression, on obtient une expression satisfaisante dans l'autre langue, il n'y a pas lieu de considérer cette expression 
comme un terme. C'est simple, mais il fallait y penser. Par exemple, «homme brave », traduit mot à mot en anglais devient : brave man. Cette traduction est des plus satisfaisantes et nous en déduisons qu'il n'y a pas lieu de considérer " homme brave » comme un terme, mais comme une expression construite à partir des termes « homme» et «brave». « Brave homme», traduit mot à mot donnerait aussi brave man, or nous savons bien que ces deux expressions n'ont pas le même sens, c'est good man ou good fellow qu'il convient de dire. Nous en déduisons que «brave homme », good man et good fellow sont des termes qu'il faut traiter en tant que tels. Appliquons le procédé à «palan pneumatique à piston», la traduction mot à mot en serait piston actuated pneumatic hoist. Nous ne trouvons cette expression, pourtant correcte et appropriée, dans aucun ouvrage anglais ou américain. Par contre, l'excellent petit manuel de Matthew W. Potts, Materials-Handling Equipment, nous donne cette définition :

Air-cylinder hoist. $A$ hoist in which a direct pull is obtained by a long-stroke piston operated by compressed air in a cylinder.

Nous considérerons donc «palan pneumatique à piston» et air-cylinder hoist comme des termes. Faute de quoi, nous risquons de voir apparaître dans des traductions des expressions telles que piston actuated pneumatic hoist et \& palan à cylindre à air comprimé », qui ne sont pas utilisées et dénonceraient une traduction lourde et maladroite.

Où trouvons-nous les termes? Dans les dictionnaires, pour ceux qui comportent un seul mot. Les dictionnaires de langue et les dictionnaires encyclopédiques nous donnent aussi quelques «mots composés», qui sont en fait des termes composés de plusieurs mots, mais ils sont loin de nous proposer tous les termes composés existants. Les dictionnaires techniques spécialisés sont plus généreux à cet égard. Mais les bons sont extrêmement rares, surtout en français. À titre d'exemple, mentionnons seulement deux excellents dictionnaires spécialisés : le Dictionnaire français de médecine et de biologie, de A. Manuila, L. Manuila, M. Nicole et $\mathrm{H}$. Lambert (connu comme le Manuila), et « a dictionary of mining, mineral and related terms», (U.S. Department of the Interior). Dans le domaine médical, le Dorland répond au Manuila. Mais c'est une exception; le dictionary of mining, mineral and related terms, l'International Dictionary of Applied Mathematics, l'International Dictionary of Physics and Electronics, le Glossary of Geology, pour ne citer qu'eux, n'ont pas d'équivalents en français, dans leurs domaines respectifs. Le terminologue doit donc se mettre en chasse, de façon systématique ou à la billebaude, pour trouver ses termes. Il consultera, bien sûr, les dictionnaires de langue, les dictionnaires encyclopédiques et les dictionnaires spécialisés, mais aussi les encyclopédies, les traités, les manuels d'enseignement, les bons ouvrages de vulgarisation, les revues sérieuses. Mais cela ne suffit pas toujours, il faut penser aux catalogues commerciaux, aux prospectus, aux visites d'usines, à la fréquentation des foires et expositions internationales, des congrès et séminaires, etc.

Il est d'ailleurs beaucoup plus difficile d'établir un dictionnaire terminologique qu'un dictionnaire de langue (ou dictionnaire de mots). Pour ce dernier en effet, le plus gros du travail préliminaire (saisie des mots et extraction des contextes) est 
effectué par ordinateur. Les tâches ultérieures (corrélation des contextes et mesure des fréquences) sont également du ressort de l'ordinateur et le lexicographe humain (coûteux et lent) travaille sur de la matière déjà traitée : contrôle des données, différenciation des acceptions, rédaction des définitions. Dans le cas d'un dictionnaire terminologique, nous avons vu qu'il était difficile de reconnaître les vrais termes, à fortiori les termes propres. Nous avons dit qu'il fallait, le plus souvent, avoir recours à une "ficelle du métier», au coup de main d'un terminologue en chair et en os, qu'il est pratiquement impossible de traduire en programme. C'est donc du tout-venant, de la matière brute, que doivent d'abord traiter les terminologues. L'ordinateur, dont les plus chanceux disposent, n'a plus alors qu'à effectuer un travail de classement sur de la matière déjà traitée.

Qui fabrique mots, termes et expressions ? C'est naturellement au rédacteur (auteur original ou traducteur) qu'incombe la tâche de fabriquer, selon ses besoins, des expressions. Nous avons vu, en effet, qu'il était impossible de tenir registre des innombrables expressions qu'on peut former avec les mots d'un vocabulaire. C'est ce qui fait la richesse d'une langue : l'infinie variété des expressions, la diversité chatoyante des tournures. S'inspirant du style des bons auteurs, le rédacteur aura soin cependant de se renouveler constamment, d'oublier les expressions toutes faites, les locutions usées, les clichés banals. Il inventera sans crainte de n'être pas compris, car une expression bien choisie se suffit à elle-même et n'a pas besoin de référence. Si les mots qui la composent sont compréhensibles, le tour selon lequel ils sont agencés exposera sans équivoque la pensée de l'auteur.

Faut-il fabriquer des mots ? Qui peut le faire? Les mots que nous connaissons ne nous sont pas tombés du ciel. Ils ont bien été proposés, inventés, fabriqués par quelqu'un. Pour certains, les dictionnaires nous donnent même le nom de l'inventeur. Des notions dont nous ne soupçonnions guère l'existence s'imposent maintenant à nous. Il faudra bien les nommer, car nous ne pouvons pas ressasser leurs descriptions ni abuser des périphrases. Mais « un mot qu'on invente ne signifie rien ${ }^{1} \gg$. Comment sortir de ce dilemme ? Car il est bien vrai qu'il faut inventer des mots et il est non moins vrai qu'un mot qui vient d'être créé, n'étant connu que de son inventeur, ne peut avoir de sens pour les autres usagers de la langue. La seule façon de s'en tirer consiste à respecter scrupuleusement ce principe fondamental : seul l'inventeur (au sens de découvreur) d'une notion peut inventer le mot pour la désigner. Celui qui découvre une notion en fait part à ses contemporains et à la postérité, en la décrivant ou en l'expliquant. Il est souvent cormmode, pour ce faire, de lui donner un nom, inventé plus ou moins arbitrairement, et qui, défini par la description donnée, pourra être ensuite utilisé en toute sécurité. Le terminologue, qui doit trouver le nom de notions existantes, ne peut pas, du moins dans l'exercice de son art, en inventer ou découvrir de nouvelles. Il ne peut donc pas, ne doit pas, inventer des mots.

Peut-il fabriquer des termes? Oui, dans certains cas très rares. Si on lui demande de nommer dans une langue, une notion qu'on lui désigne dans une autre

1. Turgot, * Tableau philosophique des progrès successifs de l'esprit humain ". (Nous pourrions ajouter : un mot qu'on adopte ou qu'on emprunte vaut ce pour quoi on
l'accepte, qu'on nomme pour cette raison son acception.) 
langue ou qu'on lui décrit, et qu'il ne trouve aucun terme attesté, il peut proposer un terme de son invention, à condition d'utiliser des mots existants, combinés de façon à suggérer sans équivoque, d'une manière précise et concise, la notion considérée. Ainsi, si «palan pneumatique à piston » n'existait pas, il faudrait l'inventer. Qui sait? Ce terme est peut-être dû à un terminologue ou à un lexicographe. Certains accordent même au terminologue le privilège, fort dangereux, de former des dérivés à partir de mots attestés, en respectant certaines règles morphologiques et sémantiques. Nous nous garderons bien de nous lancer dans une polémique à ce sujet.

La méthode du discours : notre titre était-il trompeur? En fait, nous avons surtout discuté de certains éléments du discours, nous avons parlé de procédés, mais nous n'avons pas proposé une méthode, infaillible et bien définie, de construction du discours, une martingale imbattable. Une telle méthode n'existe probablement pas. Ce qui compte, c'est de savoir ce que nous voulons dire, de bien connaître la structure et le fonctionnement des éléments dont nous disposons pour le dire, de savoir manœuvrer avec aisance et facilité ces outils merveilleux que sont les langues vivantes, nos langues, en particulier notre langue. Pour le reste, c'est une simple question de bon sens et d'inspiration. Traducteurs n'en manquent point.

HeNRI DUMAS 